\title{
The Current Status of EMG Signals on Kinematics Needed for Precise Online Myoelectric Control and the Development Direction
}

\author{
Tong Kang ${ }^{1}$ \\ ${ }^{1}$ Department: Faculty of Dentistry, Oral \& Craniofacial Sciences, King’s College London, London, SE1 9RT, the United Kingdom
}

\begin{abstract}
Widely accepted idea is the prosthetic control problem could be regarded as the pattern recognition problem. The prosthetic control means there are several differences such as distinguishable electric signals between different activation of muscle. However, this conventional method could not provide proper control of the artificial limbs. Kinematics behavior is continuous and needs the coordination of multiple physiological degrees of freedom (DOF) among various joints. Currently, a huge challenge is achieving precise, coherent and elegant coordination protheses which needs many DOFs to rehabilitation of patients with limb deficiency. This article analyzed the principles of control of bionic limbs from the aspect of EMG and traditional pattern recognition. According to the research results, the following conclusions can be given. Since the quantum amplitudes are complex numbers generally, different parameter should be included and analyzed together during the quantum information processing. Besides, the quantum control scheme could be combined with the classic one. What is more, other sensor modes should be applied for robust control instead of the EMG signal only.
\end{abstract}

\section{Introduction}

Generally, amputees will apply the myoelectric protheses to regain the function of kinematics. The surface electromyographic(sEMG) control the muscular contractions which contains cerebral motile information. Invasive Brain-Machine Interface (BMI) obtains the relationships between actual movements and neural signals which are recorded from cerebral cortex by signal processing technology, and provides users with communication and control channels to communicate with the outside world directly. Myocontrol signals are wildly used in assistive technology such as protheses and the coordination of multiple physiological degrees of freedom (DOF) across several joints. The natural motion of a limb is usually a joint motion in which these degrees of freedom are proportional at the same time. However, the number of motion controller in a pattern classification-based controller is limited. In fact, there is no pattern classification-based methods currently could propose motor signals more than two different types categories of simultaneously. Therefore, documenting reliable, accurate and instant motor neuron signals is a complicated but valuable questions. The surface electromyography (EMG) has been one of the most available methods interfacing between the patients and devices without tresis vulnus to attain the biofeedback [1].

In this review, the acquisition method of EMG signal is sorted out, and the factors that influence the surface
EMG signal, the existing problems and the contents of possible improvement methods are also discussed.

\section{Analysis}

\subsection{Possibilities of recovery of Kinematics Functions}

Prostheses as the most immediate solution for amputees. Bionic limbs establish an interface between biological residuum and an electronic device, not only controlling prosthetic movement but also providing accurate and realtime biofeedback. According to the implant used, prosthetic limbs could be divided into three main groups: targeted muscular reinnervation (TMR), direct muscle interfacing (DMI) and direct nerve interfacing (DNI). Principles of these three patterns will be represented respectively. Transferring the residual nerves from amputated limbs to available muscles is called TMR. Then, the electronic signals of muscle contractions were recorded via implants which are in body of patients and actuate the movement of prothesis. Finally, the function could be achieved through the implantable electrodes which receive signals from prosthetic sensors. Kinematic sensation will be improved. 


\subsection{EMG as neural signals \& Myoelectric control strategies}

The electrical signals produced during muscle contractions are collected, also called electromyography (EMG). For several decades, the scientists investigated that surface EMG (sEMG) is one kind of non-invasive method interfacing with related medical devices, such as the biofeedback, robotic bionic limbs, computer interfaces for hands-free. Therefore, sEMG is still mainly signal processing technology to control the activation of commercial protheses.

EMG could be regarded as neural interface or decoding the control of a phantom limb. Meanwhile, it also could be a biological amplifier of neural signal, the neural signal is also the amplifier of nerve activities [2].

EMG signals is sum of the electrical activity of muscle fibers which triggered by the activation pulses of nerve motor neuron. When increasing the nerve driving force (motor neuron number and motor neuron action potential firing frequency) to the muscle, the power of EMG monotonously increases. Generally speaking, the intensity of muscle activity is monotonously related to the intensity of nerve signals emitted by the spinal cord [3].

Mainly applied for prosthetic limbs, surface EMG signals for EMG control could decode or even process motion execution information. Prosthetic limbs could be driven through myoelectric control system. Currently, the interface could activate prosthesis monition from two different DOFs.

Therefore, when the number and frequency of motor neurons action potentials to the muscle increasing, the power of the EMG also increases. In other words, there is a positive correlation between the intensity of muscle activity and the intensity of nerve signals from the spinal cord.

However, both the number of innervated muscle fibers and surface electrodes' location will determine the total power (the property of volume conductor). Meanwhile, the extra conditions such as target task or muscle effect the performance of sEMG as well. The algorithms of myoelectric control based on these features are also influenced.

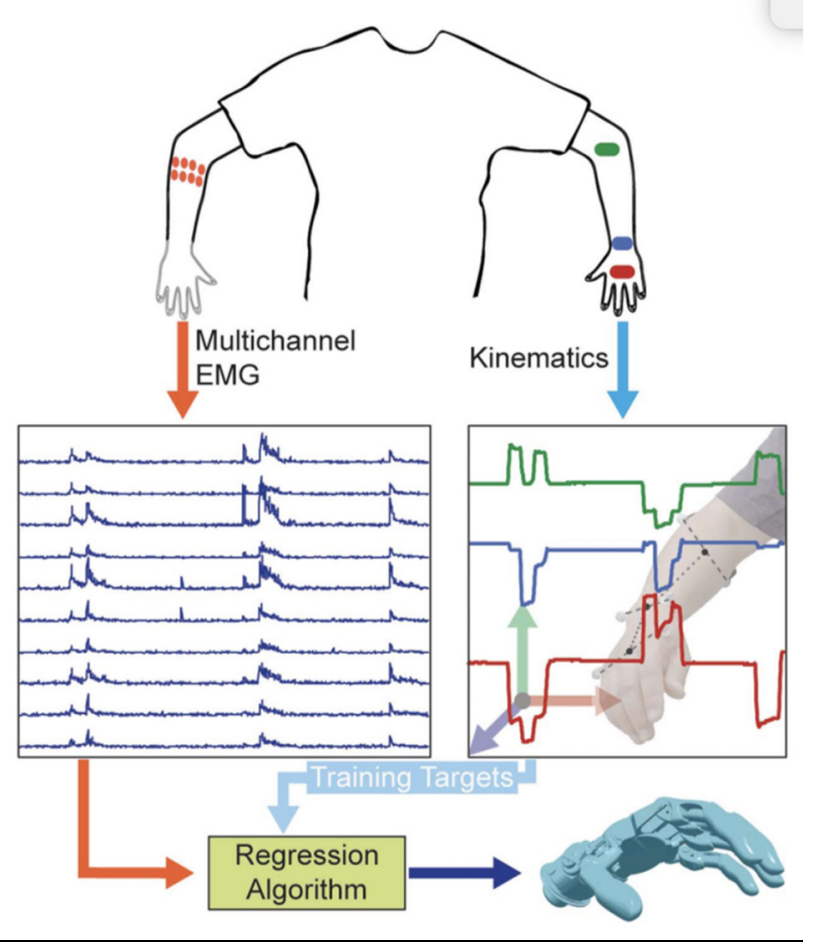

Fig 1. Example of a practical way to realize a regression approach for simultaneous and proportional control of multiple degrees of freedom. [3]

\section{Factors of influence of surface EMG}

It is well accepted that changes in electrode position or limb posture have a negative effect on the performance of myoelectric control methods.

\subsection{Crosstalk}

The distribution of action potential shapes is very different among the subjects because the differences of anatomy in various sites of motor units within the muscular tissue. The accuracy of myoelectric control algorithms is influenced greatly because of these reasons. For instance, the position of the electrodes or the gesture of the prosthetic limbs may all take negative effects to the signal collection. Simultaneously, the factors beyond human control are also take roles, such as muscle fatigue, patient's inner activation or the random change of the mode of implementations.

Nevertheless, the factors influencing the features of the EMG are not always negative at all [2]. When pattern recognition processing, the plenty of signals collected from multiple muscles will be fully utilized to define a concrete behavior then to predict intention which is the closest to the patients'. 
It is inevitable that muscles in adjacent areas generates electric signals which will disturb each other. This interference will be captured by electrodes sited on the target muscle, also called Crosstalk. In most cases, the crosstalk signal is synchronized on all channels of the array and corresponds in time to the end of the signal propagation on the stimulated muscle [4]. To some extent, the detected EMG signal is always regarded as the mixture of the motions of several contiguous muscles of similar size. Therefore, the crosstalk will affect the measurement of EMG signals, such as the electric signal power. But the negative effect could be ignored if the consistency of muscular activation is guaranteed. Contrarily, the error taken from EMG crosstalk should be integrated into the whole calculation to make sure certain accuracy.

\subsection{DOF}

Natural limbs achieve complicated, rapid and high power while robust movements by a number of bones, muscles and degrees of freedom (DOF). Thus, the dynamic parameter is continuous to describe the natural kinetics behaviors. However, in the controller-based classification, the number of the pattern is limit. The top one question about sEMG based myocontrol is setting a repeatability characteristic to guarantee reliability of the drive sources of multi degree of freedom (MDoF) prosthetic, mainly because non-stationarity of the electric signals. As the research shows, the most popular interference sources in EMG recording are the placement and displacement of electrodes, the muscle contraction and fatigue [5].

One of the reasons for the clinical failure of this type of EMG control is that it does not allow simultaneous and proportional control (SPC) of two or more degrees of freedom, which is a natural motion control method. Although recently, the simultaneous activation of two classes has been realized by enriching the training set, this method will become impractical when the number of degrees of freedom or motion increases.

\section{Conventional pattern recognition}

In fact, prosthetic control issues can be classified as same as the problems of pattern recognition. The pattern recognition is based on the hypothesis that there are distinguishable and repeatable signal patterns between different types of muscle activation.

The purpose of proposition of the pattern recognition(PR) is to control the multiple DOFs synchronously compared with conventional control system. Conventional pattern recognition usually provide control of 2 DOFs at the same time. According to the research, when ability of controlling multi-DOFs owned amputees, they could accomplish a series complex kinematics behavior better with higher accuracy, lower error frequency and faster speed [6]. Obviously, improving the amount of DOF is essential existing in the pattern recognition questions.

Pattern recognition uses classifiers trained on labeled EMG samples to distinguish multiple classes of expected motion. It is unnecessary for PR to obtain the independent control sites even without crosswalk. The transfer between models could be also ignored. A variety of feature sets and classifier combinations have been proven to provide excellent classification performance, but almost all implementations only provide seamless sequential control of multiple degrees of freedom. Linear discriminant analysis (LDA) using time-domain and autoregressive features of EMG signals is frequently used because they make the system easy to train and have high computational efficiency.

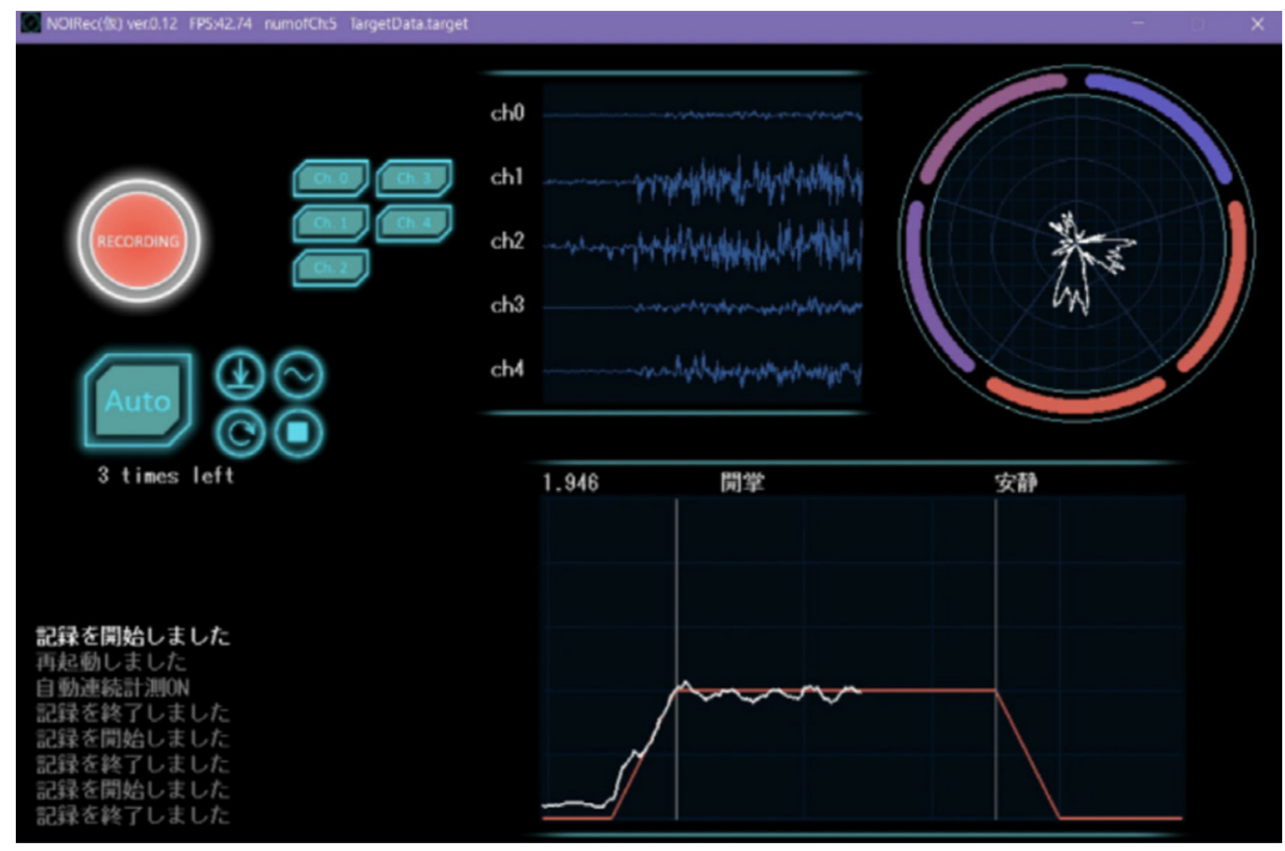

Fig 2. Interface of the developed software for collecting EMGs. [7] 


\section{New approaches}

Additional sensors may be needed to increase the functionality of prosthesis control and limit the cognitive load.

\subsection{Quantum information processing}

Quantum perceptron owns the character of strong learning and generalization ability to classify easily overlooked and overlap recognized different types of behaviors. Because of these outstanding qualities, quantum information processing could be applied in issue-solving of myoelectric control [8].

Quantum information processing could achieve concurrently and proportionally positive control of multiple DOFs just through single training of DOF data. Volunteers do not have to optimize learning stage or enlarge the scope of data collection in the simplest wrist prosthesis control scheme. The performance of the prosthesis can be improved greatly.

\subsection{Artificial neural networks (ANN)}

The neural signal extracted from surface electromyography (sEMG) could alternate conventional methods of signal collection which makes the simultaneous and proportional control(SPC) of multiDOFs more intuitive. To be more detailed, ANN in supervised regeneration method could be applied in Kinematics and Dynamics to estimate complex DOF of freedom of the contraction of wrist joint. The experiments could strongly support the efficacy of SPC no matter online or offline [9].

\section{Discussion}

It is a great potential for EMG control to improve the life quality of amputees because of the precise classification in signal recognition. This article lists some reasons for clinical limitations. Currently, mode classification of myoeletric could not control target muscle simultaneously and proportionally, the characteristic of EMG could not be recognized, the sensor modifications could not be integrated, so that the sensory feedback and continuous movement will not be achieved. These problems hinder the possibility of using this paradigm in clinical and commercial applications. During the latest few decades, the academic researches focus on refining classification accuracy. Commonly, it is essential to provide a consistent mapping between the EMG and the control signal instead of achieving extremely high accuracy estimates. This article provided two main methods which could applied in myoelectronic control to domaine the behavior of the prosthesis. The current mode classification methods mainly cannot provide synchronization and proportional control, cannot realize sensory feedback, cannot adapt to changes in EMG signal characteristics, and cannot integrate other sensor modes to achieve compound actions. These issues hinder the possibility of using this paradigm in clinical and commercial applications.

\section{Conclusion}

However, there are also something to be proved. In the period of the quantum information processing, different parameter should be included and analyzed together because quantum amplitudes are complex numbers generally. What is more, the quantum control scheme could be combined with the classic one. Considering that if only the EMG signal is used for robust control, there will be great difficulties, and it can be concluded that it is necessary to use other sensor modes. Abundant multimode inputs can not only improve control, but also lead to the development of intelligent controllers, which can operate autonomously to a certain extent, thereby reducing some of the burden on users.

\section{Acknowledgment}

I would like to thank my families, Hongfei Lee for their supports and love.

\section{References}

1. C.N. Borich, A. Fein man, M. Bertucco, N.G. Ramsy, and T.D. Sanger. Comparison of speed-accuracy tradeoff between linear and nonlinear filtering algorithms for myocontrol. Journal of Neurophysiology, 2018, 119(6), 2030-2035

2. B.P. Christie, M. Freeberg, W.D. Memberg, et al. Long-term stability of stimulating spiral nerve cuff electrodes on human peripheral nerves. J. NeuroEngineering Rehabil, 2017, 14(70). https://doi.org/10.1186/s12984-017-0285-3

3. D. Farina et al. The Extraction of Neural Information from the Surface EMG for the Control of Upper-Limb Prostheses: Emerging Avenues and Challenges. In: IEEE Transactions on Neural Systems and Rehabilitation Engineering, vol. 22, no. 4, pp. 797809, July 2014, doi: 10.1109/TNSRE.2014.2305111.

4. D. Farina, R. Merletti, B. Indino, M. Nazzaro, and M. Pozzo. Surface EMG crosstalk between knee extensor muscles: Experimental and model results. Muscle Nerve, 2002, 26, 681-695. https://doi.org/10.1002/mus.10256

5. C.N. Borish, M. Bertucco, T.D. Sanger. Effect of target distance on controllability for myocontrol. International Journal of Human-Computer Studies, 2020, vol.140, 102432, https://doi.org/10.1016/j.ijhcs.2020.102432.

6. A.J. Young, L.H. Smith, E.J. Rouse, \& L.J. Hargrove. A comparison of the real-time controllability of pattern recognition to conventional myoelectric control for discrete and simultaneous movements. Journal of Neuroengineering and Rehabilitation, 2014, 11(5). doi:http://dx.doi.org/10.1186/1743-0003-11-5

7. Y. Yamanoi, Y. Ogiri, R. Kato. EMG-based posture classification using a convolutional neural network 
for a myoelectric hand. 9. Biomedical Signal Processing and Control, 2020, vol. 55, 101574, https://doi.org/10.1016/j.bspc.2019.101574.

8. M. Siomau and N. Jiang. Myoelectric control of artificial limb inspired by quantum information processing. Published 13 February 2015 The Royal Swedish Academy of Sciences, doi:10.1088/00318949/90/3/035001

9. N. Jiang, I. Vujaklija, H. Rehbaum, B. Graimann and D. Farina, "Is Accurate Mapping of EMG Signals on Kinematics Needed for Precise Online Myoelectric Control?" in IEEE Transactions on Neural Systems and Rehabilitation Engineering, vol. 22, no. 3, pp. 549-558, May 2014, doi:

10.1109/TNSRE.2013.2287383. 